\title{
The Opportunity and Challenge of Wushu International Spread Under the Epidemic of COVID-19
}

\author{
Longfei Li \\ Faculty Of Physical Education China West Normal University, Nan Chong637002, Sichuan China, \\ e-mail:longf.acc@163.com.
}

\begin{abstract}
The unique function and healthy concept of Wushu are the basis for its inheritance and development. This paper makes an in-depth investigation and analysis on the role of Wushu in the COVID-19 by using the methods of literature and questionnaire, and finds out the opportunities and challenges of its development and dissemination, and gives the solutions to the challenges. The research holds that: in the fight against the COVID-19, Wushu once again demonstrated its unique fitness and anti-disease efficacy, which created an opportunity for the international dissemination of the concept of healthy preservation full of Chinese characteristics. However, the "twists and turns" of communication caused by its ambiguous positioning, the "integration" barrier of social behavior caused by its differentiated social psychology still pose challenges to the international communication of Wushu. Finally, it proposes to resolve the challenges in the international communication of Wushu from the aspects of accurate positioning function and emphasis on practical role, so as to promote the international communication of Wushu and contribute Chinese strength to the fight against the epidemic and maintain the health of the world's people.
\end{abstract}

Keywords: COVID-19; Martial arts; International communication; Opportunities; challenge

\section{INTRODUCTION}

"This new coronary pneumonia epidemic is a major public health emergency that has occurred in my country since the founding of New China, with the fastest spread, the widest scope of infection, and the most difficult prevention and control"-Xi Jinping. President Xi Jinping's remarks tell us that the new coronary pneumonia epidemic that started and broke out in Wuhan has had a very adverse impact on the lives and health of our people. To this end, the party and the country have used the power of the whole country to adopt various methods to "prevent" and " "Control" the epidemic. Practice has shown that before the emergence of targeted drugs, the public's own strong physique became one of the core elements of "combatting" new coronary pneumonia. Academicians, Zhong Nanshan and Li Lanjuan, also agreed this idea. Both history and science tell us that moderate physical exercise is an indispensable means of gaining a strong physique. In response to the special situation of this epidemic-the extremely infectious nature forces people to stay at home and cannot go out for exercise-and the necessarity of development of China, relevant departments and people of insight have vigorously advocated people to use national traditional sports represented by martial arts to exercise at home in order to increase their physical fitness and immunity while building national cultural confidence to fight the epidemic. Therefore, I think it is necessary to discuss the good role of martial arts (Tai Chi) in the anti-epidemic situation, show its special fitness and disease prevention effects to the international community, and find out that the epidemic situation gives great opportunities for the development of martial arts, give countermeasures to solve the problem, in order to make martial arts better serve the public health in the post-new pneumonia period, and further promote the international spread of martial arts, contribute Chinese "wisdom" to the international community to fight against the epidemic, and achieve the goal of introduction of national culture abroad.

\section{Opportunities}

Since the outbreak of the epidemic, the relevant state departments, news co-broadcast and Zhong Nanshan academicians have repeatedly stressed that due to the unique sports form and concept of martial arts, moderate practice of martial arts (Taijiquan) has a good effect and positive significance for "prevention "," anti-" and" treatment "of new crown pneumonia. About $34 \%$ of the visiting audience in the questionnaire on "The Role of Wushu in the Outbreak of the New Crown Pneumonia" answered that Wushu (Taijiquan) was successfully used to fight the new crown pneumonia. The emergence of this phenomenon undoubtedly brings new opportunities for the development of martial arts and international dissemination.

\subsection{Attractiveness: unique fitness, disease prevention and treatment effect}

The existing definition of martial arts clearly shows that martial arts is a national traditional sports that pays 
attention to both internal and external cultivation. That is to say, since the day when it was created by the Chinese people, martial arts not only require attention to external movement performance, but also emphasize internal such as Changes in breath, strength, etc. always require internal and external coordination, with "movement" and "intention" accompanying [1]. Those who have little knowledge of martial arts know that in the basic exercises of martial arts, four strikes, eight methods, and twelve types are emphasized, especially the eight methods-hands, eyes, body methods, steps, finesse, spirit, strength, and power. - In particular, it demonstrates the special requirements of internal and external integration and action in the process of martial arts practice. It can be said that there is hardly any kind of strict emphasis on "eye walk" as in martial arts in existing fitness sports. "Eye, hand, and step" show the inner essence, spirit, strength, and skill through external hands, eyes, body methods, and steps. In addition, the "internal and external triad" that is emphasized in martial arts practice also shows the special requirements for the uniformity of internal and external martial arts. For example, external triad requires hand and foot, elbow and knee, shoulder and crotch, and internal triad emphasizes the combination of heart and mind. The combination of mind and breath, and breath and strength, externally requires that hands and feet, elbows and knees, shoulders and hips must be coordinated, and internally requires that the mind be conveyed by the heart, the spirit led by the mind, and the power assisted by the gas. It also requires that the external body movements must also be consistent with the internal heart, mind, energy, and strength, and finally achieve the internal and external harmony, "movement", "intention" accompanying fitness and martial arts requirements ${ }^{[2]}$. Martial arts, a special practice method, not only strengthens the external muscle strength, bone hardness, and vascular toughness of the trainer, but also improves the internal strength and mental tension. "It has been fully shaped. It is precisely because of the unique physical fitness effect of martial arts itself, it was actively recommended by the relevant departments and individuals to the general public in this epidemic, so that the public can get "anti" and "anti" diseases through exercise. "capital". At the same time, by virtue of its special disease prevention and treatment effects, it has successfully attracted many people to participate in the exercise, such as the post-95 male nurse of the First Affiliated Hospital of Anhui Medical University to lead patients to practice Tai Chi and so on. Therefore, it is believed that the unique fitness and anti-disease effect of martial arts provides a strong impetus for its own spread.

\subsection{Internal Drive: Re-presented Health Protection Concept with Chinese Characteristics}

Since the outbreak of the new coronary pneumonia, it has continuously stimulated people to exercise their nerves to resist disease, and prompted people to exercise. It also forced the public to consider their own physical protection concepts, how to exercise and what kind of physical exercise to use It is more appropriate for the project to exercise, which has aroused the attention and thinking of many people. At this historical moment, martial arts (Tai Chi), which has unique national sports characteristics and health concepts in China, has once again entered the public's field of vision. It has been favored by the public for its natural health and long-term health protection. The research has also proved that martial arts (Tai Chi) has a practical effect on the human body to prevent and cure many diseases ${ }^{[3]}$. It is precisely because of this unique concept and function of health protection that martial arts can subtly "guide" the public in this epidemic as one of the important choices for physical exercise. History has proved that as the only country in the world's four ancient civilizations that has continued to develop to this day, its unique concept of "human" health protection plays an inestimable role in this process. The health concepts and functions possessed by martial arts are created by our working people in long-term productive labor and coexistence with nature. They are important in my country's health concepts and are outstanding representatives of my country's health protection concepts. Since the day it was created, it has always contributed its own unique strength to the healthy life of our people. For example, in the primitive society, Tao Tang's practice of using the "detumescence" "moving limbs" with martial arts prototypes to prevent and cure diseases, And in the subsequent historical development process, it gradually absorbed the essence of Confucianism, Buddhism, Taoism, and Buddhism into a unique health protection concept-with a strong body to prevent disease, that is, to cure the disease and cooperate with the treatment. The movement method and time required for illness and exercise should conform to the human body structure, regional characteristics, and the laws of daily operation. That is, the movement of the small universe of the human body should conform to the movement laws of the universe and the universe. Yamato over-preventing is not as good as yin-yang balance, etc ${ }^{[4]}$. The occurrence of this epidemic once again showed the world that martial arts are superior to other sports in terms of superior health protection concept, but also laid a certain ideological foundation for the subsequent development and spread of martial arts.

\section{CHALlenge}

Over the years, China's martial arts has always been "confused" about what to spread, how to spread, and what kind of power to spread. Although this epidemic has brought new opportunities to the international spread of martial arts, it has existed for a long time, the problem still poses a challenge to the spread of martial arts. 


\subsection{The ambiguity of its own positioning has caused the spread of "twist"}

Wushu is an excellent representative of my country's national traditional sports and national culture, an important carrier of national spirit, and an indispensable force for building national confidence. For a long time, whether it is the government living in the social management or the general public, in order to go out to realize the national culture, show the national spirit, and show the national and national image, Wushu has been used as an important carrier to promote its external communication and communication from different angles. However, martial arts are composed of technical movements, cultural thoughts, health concepts, etc., which part is used as the core positioning of its own external communication in external communication, but there are always problems. Some people pay attention to the spread of standardized technical actions ${ }^{[5]}$, such as through interviews with some so-called national martial arts team members. Most of them are "interactions" of technical actions; some scholars emphasized the dissemination from the cultural level ${ }^{6}$, when promoting martial arts to other regions or countries, they first informed the other of the culture contained in the martial arts; there were also very few scholars who mentioned the need to spread martial arts. The concept of health uses "health" as the core to promote the external communication and spread of martial arts ${ }^{[7]}$. Through the analysis of the results of the questionnaire, it is found that most of the above choices of communication positioning are the wishful thinking and practices of the main body of the communication. There are few considerations about what should be transmitted from the perspective of foreign audiences, and there is a lack of active scientific methods to understand them. The lack of understanding of the true needs of the target of communication for Chinese martial arts' behaviors has hindered the precise positioning of martial arts to the outside world and hindered the rapid spread of martial arts. Therefore, it is believed that the ambiguity of Wushu's external communication positioning has caused a "tortuous" communication.

\subsection{Differentiated social psychology hinders social behavior "integration"}

Sociologists and geographers believe that different social structures have emerged due to the different geographical environments in which the East and the West are located. China's geographical environment has created China's "differentiated" social structure. Although China has entered the so-called modern society, the social structure is still characterized by "differentiation" due to historical and cultural factors. The location of the region has prompted its social structure to appear as a "group pattern" ${ }^{[8]}$. Materialism believes that social existence determines social meaning, that is, social structure determines social psychology, and the differentiation of different social structure patterns will inevitably cause different social psychology and further cause different social behaviors ${ }^{[9]}$, which puts them in different social structure patterns The exchanges in various fields between China and the West in China have brought about original obstacles. In addition, due to some problems that have occurred in the development of China's society in the past 100 years and the adoption of methods that are not recognized by the West to promote national development, some people in Western society have developed an inherent "paranoid" mentality and stereotypes about China. The different social systems in Western countries, etc., have further exacerbated the contradiction in communication between the two parties. Wushu was created by the people in productive labor and getting along with nature in the "differentiated" structure of my country's society, and it has the characteristics of my country's social structure, psychology, and behavior. Although it has been widely used in the prevention and treatment of New Coronary Pneumonia in this epidemic, and has achieved good results, the different social psychology and behaviors caused by different social structures have caused Western society to be able to prevent and control the new crown. The recognition of pneumonia is still not high. Such a problem directly hinders the social behavior of the two parties and seriously affects the external spread of martial arts.

\section{COPING STRATEGIES}

The role of martial arts in combating new coronary pneumonia has brought new opportunities and challenges to its own communication. How to better grasp the opportunities and give appropriate strategies to meet the challenges? (Tai Chi) As an important means of combating the epidemic situation, it is worth discussing the contribution of Chinese anti-epidemic wisdom and Chinese characteristics to the international community to further promote the international spread of martial arts.

\subsection{Health function is "point", accurate communication positioning}

As one of the national traditional sports in my country, martial arts has been endowed with many functions by the promoters and researchers in the process of social promotion and dissemination, such as economy, viewing, aesthetic education and so on. However, what is the core function of martial arts has always been controversial. For example, if someone focuses on martial arts and fighting; there is a focus on inheritance and promotion of traditional culture; there are also individual emphasis on health. In order to find out the core functions of martial arts, we can't reveal them gradually from the level of practicing martial arts. We know that the process of learning martial arts technology is from the action route and direction, action specifications, strength rhythm, to the spirit. The most obvious thing that the process brings to the practitioners is 
that the muscles become stronger, the bones become tougher, and the blood vessels become more elastic. Then, the strength increases and the mental tension increases. Through analysis, it can be concluded that the most direct and core function of martial arts is the use of internal and external unity, movement and other practice methods to "send" the health of the practitioner from the outside to the inside, but this is our most in the external communication The part that is easy to ignore is also the part that is most wanted by the target of communication. For example, in the questionnaire about the role of martial arts in the new coronary pneumonia epidemic, nearly $65 \%$ of the guest audience answered their primary purpose of learning martial arts. It is physical fitness, then it is to gain fighting skills and understand Chinese culture. Therefore, it is believed that when spreading martial arts to the outside world, the core function of martial arts must be positioned to bring health to the audience, so that the "out-of-domain" audience can feel the special health function of martial arts. For example, the timely use of martial arts (Tai Chi) has been successfully used to fight the new crown pneumonia epidemic. First of all, it will use TV, Internet and other channels to let more guest audiences fully understand this matter, and show them martial arts with objective facts. Prevention and treatment of diseases, and actively guide them to participate in the use of martial arts to obtain health, through their "mouth" to spread the special health function of martial arts to their home country or region ${ }^{[10]}$, unfortunately according to the survey shows only About 34\% of the guest audience knew the role of martial arts in the fight against the epidemic. Secondly, when exchanging anti-epidemic experience with other countries outside the region, they actively "informed" the other party of the positive effects of martial arts (Tai Chi) in the fight against epidemic in China. Use the "mouth" of the other person's personnel to spread the powerful health functions of martial arts to the region, as Ambassador Fu Ying said: the Chinese story's speaking to the outside world has lifted China's own speaking, and it is necessary to learn to use the speaking of foreigners. Only when the unique health promotion characteristics of martial arts are taken as the "core point" of communication, can the audience really feel that by practicing martial arts, they can get different health experiences from other sports, so that martial arts can be truly accepted by foreign audiences To achieve the purpose of quickly spreading martial arts skills and Chinese traditional culture.

\subsection{Practice is "nuclear", dissolving social structural obstacles}

The social psychology and social behaviors caused by different social structures in China and the West have caused differences in the perceptions of the two parties on the unique health concept of martial arts, which has prevented people outside the region from using martial arts-specific health concepts to improve their own health. opportunity. How to make people outside the region realize that martial arts has a health concept different from other sports, and realize the health concept of martial arts for the benefit of all mankind deserves attention. Some scholars have said that the common practice actions of the participating subjects are the most direct and effective way for the two parties to communicate to obtain a common understanding. Marx also believes that practice is the only standard for testing truth, that is, only through practice can one party determine what needs to be communicated. "Whether it is suitable for the other party. Therefore, martial arts must focus on the practical experience of the audience in the process of external communication and communication, and actively "lead" different audiences to participate in it. For example, join a medical team similar to the foreign aid Italy, Spain and other countries to help fight the epidemic. Martial arts personnel teach local people in need to practice martial arts (Tai Chi) to prevent disease, so that more people outside the region can truly feel the special health concept and the role of disease prevention and treatment of martial arts (Tai Chi) through their own practice. That is to change their social psychology through their own practice, that is, social behavior, so that they accept martial arts from the heart, and form the concept of promoting health with martial arts, just like the initial introduction of extraterritorial sports to China, and the Chinese people at the time also showed the introduction of Western gymnastics. An attitude of resistance, but slowly accepted by the Chinese through practice, has evolved into China's original concept of sports ${ }^{[12]}$. Bourdieu and others have incisively argued that practice guides our actions. The inherent social psychology is the basis of practice, and practice produces a structure ${ }^{[12]}$, while emphasizing that the body is the center of social daily life, social culture The stable behavioral tendency system (that is, habitus) whose structure is inscribed on the body is also called stable social psychology guidance and restricts people's social practice and daily experience. Therefore, social structure and psychological structure have mutually constituted nature. That is to say, a certain social structure will form a corresponding social psychology and social behavior. Conversely, when social practice, that is, behavior and social psychology change, then the social structure will also make corresponding changes. Therefore, it is believed that the external communication of Chinese martial arts can learn from the way Western sports are introduced to me. In the process of communication, the subject needs to make its own efforts to let the audience outside the domain get a good practice experience brought by martial arts, so that it gradually accepts martial arts. In order to effectively cross the original social psychological gap, such as values, behavioral orientation, etc., dissolve the original social structure barriers, build a new inclusive social structure, and finally achieve martial arts communication

\section{CONCLUSION}

As an excellent representative of China's national traditional sports and national traditional culture, martial 
arts itself has many functions such as physical fitness, disease prevention and treatment, offensive and defensive fighting, inheriting traditional culture, and promoting regional cultural exchanges. It has always made a huge contribution to China's stable life and cultural exchanges in the long course of history. In the fight against the New Coronary Pneumonia epidemic, Wushu once again demonstrated its powerful functions to the world, such as the prevention and treatment of disease and the establishment of national confidence in anti-epidemic culture, it once again attracts people's attention and thinking at the same time. The article analyzes the opportunities and challenges that the epidemic has brought to the development and spread of martial arts through various methods such as literature and questionnaire surveys, and proposes to address the existence of communication from the aspects of accurate positioning function, focus on practical role, and rich content. The challenge is to promote the rapid and healthy external communication of martial arts, and at the same time contribute Chinese strength and Chinese wisdom to the health of the people of the world, the fight against epidemics and the establishment of a community of human destiny.

\section{Foundation Items}

This project was supported partially by Ministry of Education Humanities and Social Sciences Youth Fund Project (18JYC890015); and State Sports General Administration Sports Culture Development Center China West Normal University Sports Culture Research Base Project (19TB007); and China West Normal University Young Teacher Research Funding Project (19D011).

\section{ACKNOWLEDGMENT}

First of all, I would like to thank the Ministry of education's humanities and Social Sciences Youth Fund Project (18jyc890015), the sports culture research base project of Xihua Normal University (19tb007) of the sports culture development center of the General Administration of sport of China, and the scientific research support of young teachers of Xihua normal University. Secondly, I would like to thank Liu Xiaoling, a high school teacher, for their guidance and help in translating this paper.

\section{REFERENCES}

[1] Wang Gang, Han Jinqing. Explanation of Origin:"Moving from Movement to Quiet Return"in Chinese Wushu [J]. Journal of Tianjin Institute of Physical Education,2018,33(2):103-109.

[2] Cao Hua, Zhao Guangsheng. Exercise and Wushu Skills Acquisition [J]. Journal of Shandong Institute of Physical Education,2014,30(2):59-62.

[3] Feng Wei. Effect of Taijiquan on Fitness in Healthy China [J]. Journal of Guangzhou Institute of Physical Education,2019,39(1):87-90.

[4] Wang Lin, Chun Zaiqing. Theoretical Logic and Practical Ideas of Health Communication in Chinese Wushu [J].Journal of Wuhan Institute of Physical Education,2013,47(4):62-67.

[5] Li Shoupei, Guo Yucheng. A Study on Standardization of Traditional Wushu Technical System and Its Countermeasures [J].Sports Science,2015,35(2):81-89. Guo Yucheng, Li Shoupei. A study on the orientation of Wushu's construction of Chinese national image $[\mathrm{J}]$. Journal of Beijing University of Physical Education,2013,36(9):9-18.

[6] Lu Shaojun. The Strategic Transformation of Chinese "Culture Going Out" under the "Belt and Road" Initiative -Taking the 3.0 Age of Wushu International Promotion as an Example [J]. Journal of Beijing University of Physical Education,2018,41(6):1-6.

[7] Fei Xiaotong. Country China [M]. Beijing: People's Publishing House ,2015:25-41.

[8] High strength. A Study on Bourdieu's Sociology of Sports [M]. Beijing: Intellectual Property Press ,2014:11.

[9] Wang Gang, Hou Liankui, Jiang Limin. Chinese Wushu: An Adult Learning [J]. Journal of Wuhan Institute of Physical Education,2019,53(11):57-63,100.

[10] Liu Sixen. A Study on the Spread of Modern Western Sports in China [J]. Sports Culture Guide,2014,(2):182-184.

[11] Bourdieu, Hua Kant. Guidance on reflective sociology [M].] Beijing: Commercial Press,2015:167.

[12] Bourdieu. Distinction: Social Critique of Judgment [M]. Shanghai: Commercial Press,2015:270-275. 